\title{
Quantum nondemolition measurements of a qubit
}

\author{
D.V. Averin \\ Department of Physics and Astronomy, SUNY Stony Brook, Stony Brook, NY 11794-3800
}

(October 26, 2018)

\begin{abstract}
The concept of quantum nondemolition (QND) measurement is extended to coherent oscillations in an individual two-state system. Such a measurement enables direct observation of intrinsic spectrum of these oscillations avoiding the detector-induced dephasing that affects the standard (non-QND) measurements. The suggested scheme can be realized in Josephson-junction qubits which combine flux and charge dynamics.
\end{abstract}

Quantum coherent oscillations in a two-state system (qubit) represent the most basic dynamic manifestation of quantum coherence between the qubit states. Motivated by potential application to quantum computation [1,2] and conceptual interest in macroscopic quantum phenomena, significant effort is devoted at present to attempts to observe and study these oscillations in individual "mesoscopic" qubits realized with Josephsonjunction systems - see, e.g., [3 [7]. One of the most direct ways of detecting the coherent oscillations in a qubit is to monitor them continuously with a weakly-coupled linear detector [8]. Spectral density of the detector output should exhibit then the spectral line at the oscillation frequency which contains information about the oscillation amplitude and decoherence rate, and has other interesting features. For example, the absolute intensity of the oscillation line demonstrates directly the quantum nature of the oscillations. It exceeds by a factor of two intensity of the classical harmonic oscillations of the same amplitude. Quantum mechanics makes larger intensity possible by combining harmonic oscillations of the probability with discrete jumps of the oscillating variable between the two states of the qubit [8].

The spectral line in the detector output, however, does not fully represent intrinsic spectral density of the oscillations. In the simplest measurement scheme the detector measures directly the oscillating coordinate, and thus tends to localize it, introducing extra dephasing in the dynamics of the oscillations. Such a "backaction dephasing" creates a fundamental limit, equal to 4 , for the signal-to-noise ratio of the measurement, i.e., the ratio of the height of the oscillation line to the output noise of the detector. This limitation makes direct measurement of the quantum coherent oscillations in an individual qubit difficult, and leads to an interesting question whether a variant of quantum nondemolition (QND) technique can be used to avoid the detector backaction and to overcome the limitation on the signal-to-noise ratio. This work suggests such a QND technique and develops its quantitative description.

QND measurement technique was proposed first for detection of weak forces acting on a harmonic oscillator in the context of the gravitational-wave antennas [9, 10], and was discussed until now in application to measurements of various realizations of harmonic oscillators - see, e.g., [11, 12]. Here the concept of a QND measurement is extended to the two-state system. In general, the QND measurement is realized when a quantum system is coupled to a measuring detector through an operator (the measured observable) that represents at least an approximate integral of motion. In this case, the backaction by the detector which increases fluctuations and uncertainty in the variables not commuting with the measured observable does not couple back into its evolution. Such a "decoupling" of backaction makes it possible to measure the system continuously without significantly perturbing it.

This discussion implies that specific scheme of the QND measurement of a two-state system should depend on the main part of the system Hamiltonian. In what follows, we consider the case of unbiased two-state system that is the most advantageous for the quantum coherent oscillations. In the basis of the two states of the oscillating variable $\hat{x} \propto \sigma_{z}$ (e.g., charge or flux states in the case of charge [3, 4] or flux [5] [7] qubits, respectively) the Hamiltonian of the system is then

$$
H=-\frac{1}{2} \Delta \sigma_{x}
$$

Here and below $\sigma$ 's denote Pauli matrices, and $-\Delta / 2$ is the amplitude of tunnel coupling between the basis states. The basic idea behind the QND measurement of such a system is illustrated in Fig. 1. In the spin-1/2 representation of the two-state system (1), its dynamics can be seen as rotation with frequency $\Delta$ in the $y-z$ plane. To perform the QND measurement, the direction along which the spin is measured should follow as closely as possible the system rotation. This is achieved if the measurement direction rotates with frequency $\Omega \simeq \Delta$. (Since the phase of the oscillation of a spin $1 / 2$ can not maintain any semiclassical dynamics, there is no question of the phase relation between the two rotations.) Thus, one can suggest the following Hamiltonian for the QND qubit measurement: 


$$
H=-\frac{1}{2} \Delta \sigma_{x}-\frac{1}{2}\left(\cos \Omega t \sigma_{z}+\sin \Omega t \sigma_{y}\right) f+H_{0} .
$$

Here $H_{0}$ is the Hamiltonian of the detector which is coupled to the qubit via the force $f$, and to simplify notations all coupling constants are included in the definition of $f$.

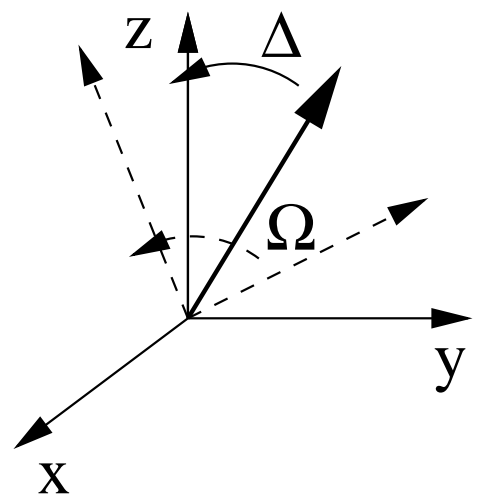

FIG. 1. Spin representation of the QND measurement of the quantum coherent oscillations of a qubit. The oscillations are represented as a spin rotation in the $z-y$ plane with frequency $\Delta$. QND measurement is realized if the measurement frame (dashed lines) rotates with frequency $\Omega \simeq \Delta$.

The Hamiltonian (2) of the QND qubit measurement is different from that of the non-QND measurement studied so far in theory [1,2,8,13,16] and used in experiments [3] in the form of the qubit-detector coupling. The coupling term in (2) should be contrasted with $-\sigma_{z} f / 2$ in the non-QND case when the detector is coupled directly to the oscillating variable. Apart from this difference, the two situations should be similar, and the detector properties in (2) can be taken to be the same as in the previously studied non-QND case: the detector is linear, with frequency-independent (in the frequency range given by the tunnel amplitude $\Delta$ ) response coefficient $\lambda$. These assumptions imply that the force $f$ can be viewed as random classical $\delta$-correlated variable with the correlation function

$$
\langle f(t+\tau) f(t)\rangle_{0}=2 \pi S_{f} \delta(\tau),
$$

where the average $\langle\ldots\rangle_{0}$ is taken over the detector density matrix, and $S_{f}$ is the constant low-frequency part of the spectral density of $f$, i.e., the detector backaction noise. For more detailed discussion of this detector model see [13]. Under the same assumptions, the correlation function of the detector response to the oscillations is:

$$
K(\tau)=\frac{\lambda^{2}}{8}[\langle c(t) c(t+\tau)+c(t+\tau) c(t)\rangle],
$$

where $c \equiv \cos \Omega t \sigma_{z}+\sin \Omega t \sigma_{y}$ is the operator of qubitdetector coupling and the average is now taken both over the detector and qubit density matrices. The time dependence of $c$ 's in eq. (4) combines explicit time dependence in their definition and time evolution with the Hamiltonian (2).
To calculate the correlator (幽 we notice that the explicit time dependence of the coupling operator $c$ can be written as

$$
c=e^{i \Omega \sigma_{x} t / 2} \sigma_{z} e^{-i \Omega \sigma_{x} t / 2} .
$$

This relation follows directly from properties of the Pauli matrices and expresses quantitatively the notion of "rotation of the measurement direction" in Fig. 1. Using this relation one can check that the time evolution operator $S$ associated with the Hamiltonian (2) has a simple form in the rotating measurement frame:

$$
\begin{gathered}
S\left(t_{1}, t_{2}\right)=T \exp \left\{-i \int_{t_{2}}^{t_{1}} d t^{\prime} H\left(t^{\prime}\right)\right\} \\
=e^{i \Omega \sigma_{x}\left(t_{1}-t_{2}\right) / 2} e^{-i H^{\prime}\left(t_{1}-t_{2}\right)},
\end{gathered}
$$

where $H^{\prime}$ is an effective Hamiltonian of the system in the rotating frame:

$$
H^{\prime}=-\frac{1}{2}(\Delta-\Omega) \sigma_{x}-\frac{1}{2} \sigma_{z} f+H_{0} .
$$

Using the fact (demonstrated more explicitly below) that the correlator (4) should be independent of the initial density matrix $\rho$ of the qubit, we can take $\rho$ in the simplest form $\rho=1 / 2$. Equations (5) and (6) allow us then to reduce the correlator (4i) to the following form:

$$
K(\tau)=\frac{\lambda^{2}}{4} \operatorname{Re}\left\langle\sigma_{z} \sigma_{z}(\tau)\right\rangle, \quad \sigma_{z}(\tau)=e^{i H^{\prime} \tau} \sigma_{z} e^{-i H^{\prime} \tau} .
$$

To find the average of the operator $\sigma(\tau)$ in (8) over the detector backaction noise $f$, it is convenient to start with the Heisenberg equations for $\sigma_{z}(\tau)$ with the Hamiltonian $H^{\prime}$. Averaging the resulting equations for the matrix elements $\sigma_{i j}$ of $\sigma_{z}(\tau)$ with the help of the correlator (3) we get:

$$
\dot{\sigma}_{11}=i \delta\left(\sigma_{12}-\sigma_{21}\right) / 2, \quad \dot{\sigma}_{12}=i \delta\left(\sigma_{11}-\sigma_{22}\right) / 2-\Gamma \sigma_{12},
$$

and $\dot{\sigma}_{22}=-\dot{\sigma}_{11}$. Here $\delta \equiv \Delta-\Omega$, and $\Gamma=\pi S_{f}$ is the rate of backaction dephasing of the oscillations by the detector.

As the next step, we need to take into account environment-induced energy relaxation/dephasing that affects the qubit in addition to the detector backaction. Assuming that interaction with the environment is weak, so that the characteristic relaxation rate is much smaller than the tunnel amplitude $\Delta$, we can simply add the corresponding terms in the equation (9) for $\sigma_{z}(\tau)$. It is more convenient to do this not directly in the basis of eigenstates of the oscillating coordinate used in (9), but in the basis of energy eigenstates of the qubit. Transforming eq. (9) into the energy basis (in the spin notations, the transformation is the $\pi / 2$ rotation around the $y$ axis: 
$\left.\sigma_{z} \rightarrow \sigma_{x}, \sigma_{x} \rightarrow-\sigma_{z}\right)$ and adding the term responsible for the environmental relaxation, we obtain the following equation for the off-diagonal matrix element of the operator $s$ of the oscillating variable (given by $\sigma_{z}(\tau)$ in the original coordinate basis):

$$
\dot{s}_{12}=i \delta s_{12}-\Gamma\left(s_{12}-s_{21}\right) / 2-\Gamma_{e} s_{12} .
$$

To find the correlator (8) we need to solve the evolution equations for $s$ with the initial conditions $s_{12}=1$ and the diagonal elements of $s$ equal to zero. Equations for the diagonal elements show then that they remain zero at all $\tau$, and $s_{12}(\tau)(10)$ determines the correlator completely. This means that the real part of the product $s s(\tau)$ is proportional to the unity matrix, and the correlator (8) is independent of initial qubit density matrix. We also note that the different form of the terms describing detectorand environment-induced dephasing in eq. (10) is due to the QND nature of the detector-qubit coupling, and that while the environmental relaxation rate $\Gamma_{e}$ was assumed to be much smaller than $\Delta$, both $\Gamma_{e}$ and $\Gamma$ can be larger than the detuning $\delta$.

Solving eq. (10) with the initial condition $s_{12}=1$ and substituting the solution in (8) we obtain the correlation function of the detector response to the qubit:

$$
K(\tau)=\frac{\lambda^{2}}{4} e^{-\left(\Gamma / 2+\Gamma_{e}\right) \tau}\left[\cosh D t+\frac{\Gamma}{2 D} \sinh D t\right],
$$

where $D \equiv\left(\Gamma^{2} / 4-\delta^{2}\right)^{1 / 2}$. The correlation function (11) determines the spectral density $(1 / \pi) \int d \tau K(\tau) \cos \omega \tau$ of the detector output. Including the constant output noise $S_{q}$ of the detector, the spectral density is:

$$
S(\omega)=S_{q}+\frac{\lambda^{2}}{4 \pi} \frac{\left(\Gamma+\Gamma_{e}\right)\left(\Gamma^{2}+\Gamma \Gamma_{e}+\delta^{2}\right)+\Gamma_{e} \omega^{2}}{\left(\omega^{2}-\delta^{2}+\Gamma^{2}+\Gamma \Gamma_{e}\right)^{2}+\omega^{2}\left(\Gamma+2 \Gamma_{e}\right)^{2}} .
$$

If the detuning $\delta$ is much larger than the dephasing rates, the spectral density reduces to the two Lorentzian peaks at $\omega= \pm \delta$. In the vicinity of the positive-frequency peak, the spectral density can be written as

$$
S(\omega)=S_{q}+\frac{\lambda^{2}}{8 \pi} \frac{\Gamma_{e}+\Gamma / 2}{(\omega-\delta)^{2}+\left(\Gamma_{e}+\Gamma / 2\right)^{2}} .
$$

This expression shows that for large detuning, the spectral density is close to the one obtained in the usual, non-QND measurement [8,13]. The only difference is the frequency shift of the spectral peak from intrinsic oscillation frequency $\Delta$ by the frequency $\Omega$ of rotation of the measurement frame. The main goal of the QND technique, avoiding the detector backaction, is not reached in this regime. The backaction dephasing broadens the oscillation spectral line, and limits the height of the oscillation peak relative to the background set by the detector output noise. Indeed, eq. (13) shows that even for
$\Gamma_{e}=0$, the maximum height $S_{\max }$ of the oscillation peak is $S_{\max }=\lambda^{2} / 4 \pi \Gamma$, and the limitation on the response coefficient of the detector from the linear-response theory, $\lambda \leq 4 \pi S_{f} S_{q}[13$, shows that the peak is limited as in the non-QND measurement: $S_{\max } / S_{q} \leq 4$.

The situation, however, changes, if the frequency of rotation of the measurement frame matches the oscillation frequency more accurately, so that $\delta \ll \Gamma$. The oscillation line is shifted then to zero frequency, and the lineshape is:

$$
S(\omega)=S_{q}+\frac{\lambda^{2}}{2 \pi} \frac{\Gamma_{e}+\gamma}{\omega^{2}+\left(\Gamma_{e}+\gamma\right)^{2}}, \quad \gamma \equiv \delta^{2} / \Gamma .
$$

Qualitatively, $\gamma$ in this equation is the rate of rare jumps of spin in the rotating measurement frame between the positive and negative measurement direction, and we see that the spectral line is now broadened not directly by the backaction dephasing but by these rare jumps. The most important feature of these jumps is that the rate $\gamma$ vanishes together with the detuning $\delta$, and the spectrum (14) of the detector output reproduces then intrinsic linewidth of the oscillation unaffected by the backaction dephasing. Therefore, "rotating" measurement (2) with frequency $\Omega$ equal to the tunnel frequency $\Delta$ avoids the detector backaction and realizes the QND measurement of the quantum coherent oscillations in a two-state system. Other methods of avoiding the backaction dephasing are based on control of the oscillations via the feedback [17]. These methods, however, do not represent real "measurement" but rather creation of the oscillations.

The QND technique is required to measure the intrinsic spectral line of the oscillations in a qubit. Such an intrinsic oscillation line is typically found in calculations that implicitly assume that the spectral density can be measured without disturbance from the detector even in quantum mechanics - see, e.g., 18]. As we saw above, in the case of mesoscopic qubits this assumption is not obvious, and non-trivial measurement schemes are required to observe intrinsic oscillation spectrum. Without these schemes, the detector backaction affects the spectrum at least within the linear detection approach appropriate for typical mesoscopic detectors.

In mesoscopic qubits, for which the question of measurement of an individual qubit is particularly relevant, the necessity to measure two non-commuting qubit operators to implement the QND measurement presents a non-trivial requirement since only one (basis-forming) observable typically has clear physical realization. A system where this requirement can be satisfied is the Josephson-junction qubit that combines charge and flux dynamics. The simplest version of such a qubit is the "charge-controlled SQUID": two junctions included in a superconducting loop with inductance $L$, with the island between the junctions that has small electric capacitance $C$ (Fig. 2) 19]. External magnetic flux $\Phi_{e}$ is applied to 
the loop, and a gate electrode induces charge $q$ on the middle island.

\section{qubit electrometer}

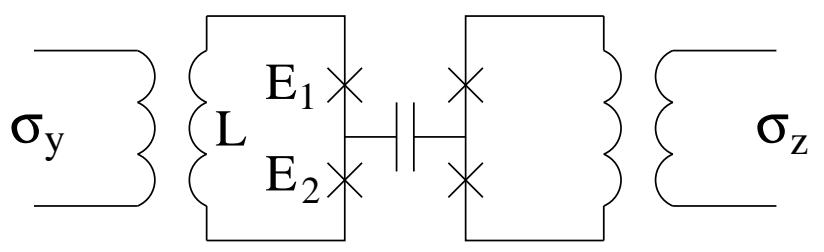

FIG. 2. Schematic of the Josephson-junction qubit structure that enables measurements of the two non-commuting observables of the qubit, $\sigma_{z}$ and $\sigma_{y}$, as required in the QND Hamiltonian (2). For discussion see text.

Depending on the parameter values, this system can act as a charge or flux qubit. To be specific, we consider here the situation when the junction coupling energies $E_{1,2}$ are small in comparison to the island charging en$\operatorname{ergy}(2 e)^{2} / 2 C$, and the inductance $L$ is also small. Then, dynamics of charge $2 e n$ on the middle island dominates the system, and for $q \simeq e$ is reduced to the two-state dynamics with the basis states $n=0$ and $n=1$. If the frequency of the "plasma" oscillations of the flux $\Phi$ in the loop $L$ around the external flux $\Phi_{e}$ is much larger than the energies of the charge qubit, the oscillations effectively decouple from the charge dynamics, and the charge part of the system Hamiltonian reduces then to:

$$
H=\frac{e(e-q)}{C} \sigma_{z}-E_{+} \cos \varphi_{e} \sigma_{x}+E_{-} \sin \varphi_{e} \sigma_{y} .
$$

Here $E_{ \pm} \equiv\left(E_{1} \pm E_{2}\right) / 2$, Pauli matrices act in the basis of the two charge states $n=0$ and $n=1$, and $\varphi_{e} \equiv e \Phi_{e} / \hbar$.

The flux part of the system still plays important role, since the tunneling of Cooper pairs in the charge qubit (15) produces current in the loop:

$$
I=\frac{1}{2}\left(I_{+} \sin \varphi_{e} \sigma_{x}+I_{-} \cos \varphi_{e} \sigma_{y}\right),
$$

where $I_{j}=2 e E_{j} / \hbar$ are the junction critical currents, and $I_{ \pm} \equiv\left(I_{1} \pm I_{2}\right) / 2$. (The last equation assumes, for simplicity, that the junction capacitances are equal.) For finite loop inductance $L$, the current (16) creates small variations of the flux $\Phi$ through the loop that can be detected by external system, and by monitoring these variations one can measure either $\sigma_{x}$ or $\sigma_{y}$ component of the charge qubit (15). The possibility to measure all observables of an individual mesoscopic qubit provided by the qubit (15) can be important for many different purposes.

When $\Phi_{e}=0$ and $q=e$, the qubit (15) provides the necessary elements for the QND measurement discussed above. In this case, eq. (15) reduces to the unbiased Hamiltonian (1) with the tunnel amplitude $E_{+}$, and the current $I=\left(I_{-} / 2\right) \sigma_{y}$ in the inductance $L$ represents the $\sigma_{y}$ component of the qubit dynamics. The $\sigma_{z}$ component can obviously be measured through the charge on the middle electrode of the qubit. The final step in realization of the QND Hamiltonian (2) is to convert both measurement components into one physical form (e.g., charge or flux) and apply (with sine- and cosine-modulated coupling strength) to one detector. One way of achieving this is to convert the charge signal $\left(\sigma_{z}\right)$ into the flux form by another charge-controlled SQUID operated in the regime of Bloch electrometer [20] (Fig. 2), when all internal frequencies of the electrometer are much larger than the frequency $E_{+}$of the charge signal. The signal modulates then the quasistationary critical current of the double junction system and, as a result, changes the current and the flux in the electrometer loop. Once the two signals are in the flux form, the subsequent steps can be realized using known Josephson-junction circuits: modulated flux transformers and magnetometer.

To summarize, it is possible to design the QND technique for measurement of the quantum coherent oscillations in an individual two-state system. The technique avoids the detector backaction, and overcomes the limitation on the signal-to-noise ratio of the measurement of the spectral density of the oscillations imposed by the backaction dephasing.

This work was supported in part by the AFOSR, and by the NSA and ARDA under the ARO contract. The author would like to thank K.K. Likharev and V.K. Semenov for useful discussions.

[1] D.V. Averin, Fortschrit. der Physik 48, 1055 (2000).

[2] Yu. Makhlin, G. Schön, and A. Shnirman, Rev. Mod. Phys. 73, 357 (2001).

[3] Y. Nakamura, Yu.A. Pashkin, and J.S. Tsai, Nature 398, 786 (1999).

[4] V. Bouchiat, D. Vion, P. Joyez, D. Esteve, and M.H. Devoret, J. Supercond. 12, 789 (1999).

[5] J.R. Friedman, V. Patel, W. Chen, S.K. Tolpygo, and J.E. Lukens, Nature 406, 43 (2000).

[6] C.H. van der Wal, A.C.J. ter Haar, F.K. Wilhelm, R.N. Schouten, C. Harmans, T.P. Orlando, S. Lloyd, and J.E. Mooij, Science 290, 773 (2000).

[7] S.Y. Han, Y. Yu, X. Chu, S.I. Chu, and Z. Wang, Science 293, 1457 (2001).

[8] A.N. Korotkov and D.V. Averin, Phys. Rev. B 64, 165310 (2001).

[9] C.M. Caves, K.S. Thorne, R.W.P. Drever, V.D. Sandberg, and M. Zimmermann, Rev. Mod. Phys. 52, 341 (1980).

[10] V.B. Braginsky and F.Ya. Khalili, Rev. Mod. Phys. 68, 1 (1996).

[11] P. Grangier, J. A. Levenson, and J.-P. Poizat, Nature 
396, 537 (1998).

[12] S. Peil and G. Gabrielse, Phys. Rev. Lett. 83, 1287 (1999).

[13] D.V. Averin, in: "Exploring the Quantum-Classical Frontier: Recent Advances in Macroscopic and Mesoscopic Quantum Phenomena", Eds. J.R. Friedman and S. Han, to be published; cond-mat/0004364.

[14] Yu. Makhlin, G. Schön, and A. Shnirman, Phys. Rev. Lett. 85, 4578 (2000).

[15] M.H. Devoret and R.J. Schoelkopf, Nature 406, 1039 (2000).

[16] A. Aassime, G. Johansson, G. Wendin, R.J. Schoelkopf, and P. Delsing, Phys. Rev. Lett. 86, 3376 (2001).

[17] R. Ruskov and A.N. Korotkov, cond-mat/0107280.

[18] M.-S. Choi, F. Plastina, and R. Fazio, Phys. Rev. Lett. 87, 116601 (2001).

[19] J.R. Friedman and D.V. Averin, Phys. Rev. Lett. 88, 050403 (2002).

[20] A.B. Zorin, Phys. Rev. Lett. 86, 3388 (2001). 